\title{
Malnutrition as predictor of poor outcome after total hip arthroplasty
}

\author{
Sandra Eminovic ${ }^{1} \cdot$ Gabor Vincze $^{1}$ (D) $\cdot$ Doris Eglseer ${ }^{2} \cdot$ Regina Riedl $^{3} \cdot$ Patrick Sadoghi $^{1} \cdot$ Andreas Leithner $^{1}$. \\ Gerwin A. Bernhardt ${ }^{1}$
}

Received: 12 November 2020 / Accepted: 18 November 2020 / Published online: 26 November 2020

(C) The Author(s) 2020

\begin{abstract}
Introduction The aim of this study was to assess the prevalence of protein energy malnutrition (PEM) and correlation with poor post-operative outcome in the elderly undergoing primary total hip arthroplasty (THA).

Hypothesis Patients with PEM would have inferior post-operative outcome after THA.

Materials and method We retrospectively evaluated the nutritional status of 220 hospitalized patients undergoing THA, 65 years and older. PEM was assessed using serum albumin and total lymphocyte count (TLC). Studied outcome parameters were length of pre-operative and post-operative stay, complications up to six months after surgery and 12-month mortality. Clinical and demographic data were retrieved from medical records from the hospital database.

Results The prevalence of PEM among patients undergoing THA was $12.3 \%$ (27/220). Patients with PEM were significantly older (mean age $81.3 \pm 7.0, p<0.001)$, had a lower BMI $(24.7 \pm 4.1 \mathrm{~kg} / \mathrm{m} \mathrm{2}, p=0.022)$, and showed more comorbid conditions (mean CCI $2.8 \pm 2.0, p=0.002$ ) compared with well-nourished patients (age 75.6 \pm 6.2 , BMI $26.8 \pm 4.3 \mathrm{~kg} / \mathrm{m} \mathrm{2}$, CCI: $1.7 \pm 1.7$ ). Length of pre-operative stay differed significantly $(p<0.001)$ between PEM (median 7, range 1-36 days) and non PEM (median 1, range 1-22 days). In the PEM group, 12 (44.4\%) patients had post-operative complications within six months after OP and 15 (7.8\%) patients in the non PEM group (HR $=6.3,95 \%$ CI 1.7-23.1).

Conclusion We observed a higher post-operative complication rate for malnourished patients undergoing elective THA. These results underline the importance of pre-operative nutritional assessment in the elderly. Therefore, serum albumin and TLC are valuable clinical markers of PEM and the post-operative outcome.
\end{abstract}

Keywords Malnutrition $\cdot$ PEM $\cdot$ Surgery $\cdot$ Total hip arthroplasty $\cdot$ Post-operative outcome

\author{
Abbreviations and acronyms \\ PEM Protein energy malnutrition \\ THA Total hip arthroplasty \\ TLC Total lymphocyte count
}

Gerwin A. Bernhardt

gerwin.bernhardt@medunigraz.at

1 Department of Orthopedics and Traumatology, Medical University of Graz, Auenbruggerplatz 5, 8036 Graz, Austria

2 Institute of Nursing Science, Medical University of Graz, Universitätsplatz 4, $8010 \mathrm{Graz}$, Austria

3 Institute for Medical Informatics, Statistics and Documentation, Medical University of Graz, Auenbruggerplatz 2, 8036 Graz, Austria

\section{Introduction}

Osteoarthritis (OA) is one of the most common joint disorders in the elderly with a prevalence up to $45 \%$ in general population [1]. To alleviate the consequences of $\mathrm{OA}$ as pain or disability, total joint replacement (TJR) is commonly performed. One of the most often affected joints with OA is the hip requiring total hip arthroplasty (THA) in end stage osteoarthritis.

Besides, elderly patients show a higher risk for malnutrition. For those affected, this can lead to longer hospital stays with increased morbidity and higher mortality $[2,3]$. Poor nutritional status tends to result in impaired wound healing or an increased risk of infections [4].

Successful rehabilitation after THA is determined by patients' peri-operative health status. Hospitalization represents a stressful event, and consequently, the nutritional status tends to deteriorate during the hospital stay. Several studies are demonstrating the association of poor nutritional status and adverse outcomes in surgical patients $[2,5]$. 
Definitive prevalence of malnutrition seems to be unclear, as there is no consensus on the best screening method. Various combinations of indicators have led to a range of scales. Consequently, the reported prevalence in patients undergoing elective joint arthroplasty varies widely between 8.5 and $30 \%$ [6]. Identifying the risk of adverse post-operative outcomes laboratory values may be used as a nutritional screening tool for hospitalized persons [7]. To assess protein energy malnutrition (PEM), biochemical parameters such as serum albumin and total lymphocyte count (TLC) as predictors of outcome in hip fracture patients have been used in literature [2, 3]. Malnutrition seems to be a common causative factor for hip fractures [8] as several studies demonstrated poor outcome in malnourished hip fracture patients [5, 9]. So far only a few studies indicated post-operative outcome in patients undergoing elective arthroplasty [6]. Given that total arthroplasty among older people is associated with high post-operative complications, the pre-operative nutritional status can be emphasized for patients' recovery $[2,5]$. However, the controversial impact of pre-operative nutritional status in patients undergoing THA has not fully been evaluated in the literature.

The aim of this study was therefore to assess the prevalence of PEM and to assess whether pre-operative nutritional parameters are associated with poor post-operative outcome in the elderly undergoing primary total hip arthroplasty. Our hypothesis was that patients with PEM would have inferior postoperative outcome after THA.

\section{Materials and methods}

All patients who underwent primary THA at a department of orthopaedic surgery in an 8-year period were retrospectively studied. Patients' clinical and demographic data were retrieved from medical records from the hospital database. Patients' chronic medical conditions were assessed with the Charlson comorbidity index (CCI) [10] based on the medical history. All 19 comorbidities necessary to calculate the CCI and the age of the patients were taken into account. To assess patients' pre-operative physical status, American Society of Anesthesiologists (ASA) rating of operative risk was used from medical records. Patients suitable for operation were classified as ASA categories I, II, III, and IV as used previously [11].

Serum albumin and TLC were retrieved from a computerized laboratory database. All testing was performed by the same laboratory with standardized reference values for each test. All samples were taken at admission and on the first postoperative day. Pre-operative values of $<3.5 \mathrm{~g} / \mathrm{dl}$ of serum albumin and $<1.5 \mathrm{~g} / 1$ of TLC were considered as PEM [3]. Given that OA affects mostly older individuals we decided to include patients 65 years and older. Patients without laboratory tests performed pre-operatively were excluded. Pre- operative transfusion rates were not evaluated, and we do not pre-operatively assess osteoporosis through DEXA. The included patients were divided into two groups:

Non PEM: pre-operative albumin $\geq 3.5 \mathrm{~g} / \mathrm{dl}$ or $\mathrm{TLC} \geq$ $1.5 \mathrm{~g} / 1$

PEM: pre-operative albumin $<3.5 \mathrm{~g} / \mathrm{dl}$ and TLC $<1.5 \mathrm{~g} / 1$

Time from admission to operation (pre-operative stay), length of post-operative stay, post-operative complications after three and six months, and 12-month post-operative mortality were assessed and compared between the groups. Postoperative complications were defined as any deviation of a routine post-operative hospital stay. They were classified dichotomously (present/absent) and represented at least one of the frequent short-term complications including surgical and non-surgical complications such as infections, pulmonary intricacies, impaired wound healing, or vascular complications. Post-operative complications included subsequent or repeated re-admissions that followed the initial hospitalization and could not be foreseen at the time of discharge. Also included were acute admissions to other wards while patients were hospitalized at our department.

\section{Statistical analysis}

Continuous variables are presented as mean \pm standard deviation or median and minimum, maximum, and categorical variables as frequencies and percentages. Group comparisons were performed by using Chi-square or Fishers exact test and $t$ test or Mann-Whitney U test, as appropriate. For complications six months after surgery, Kaplan-Meier curves are presented and hazard ratios (HR) with their corresponding 95\% confidence intervals (CI) were estimated using a Cox proportional hazard models including group (PEM vs. non PEM), gender, age, type of diagnosis, time to operation, pre-operative $\mathrm{C}$-reactive protein (CRP), and pre-operative hemoglobin (HB). A $p$ value of $<$ 0.05 was considered to indicate statistical significance. All $p$ values are regarded in an explorative sense. The statistical analysis was performed using the statistical software SPSS, Version 22 (SPSS Inc., Chicago, Ill).

\section{Results}

\section{Sample characteristics}

A total of 1183 patients underwent primary THA during the study period. Pre-operative serum albumin and TLC levels were available for $220(18.6 \%)$ patients. The majority of the patients were female $(n=144,65.5 \%)$ and the mean age was $76.3 \pm 6.6$ years, ranging from 65 to 95 years (Table 1 ). The 
Table 1 Demographic data and patient characteristics

\begin{tabular}{ll}
\hline Characteristics & Study group $n=220$ \\
\hline Gender & \\
Female & $144(65.5 \%)$ \\
Male & $76(34.5 \%)$ \\
Age (years) & $76.3 \pm 6.6$ \\
Diagnosis & \\
OA & $195(88.6 \%)$ \\
Hip fracture & $25(11.4 \%)$ \\
BMI $\left(\mathrm{kg} / \mathrm{m}^{2}\right), n=185$ & $26.5 \pm 4.3$ \\
CCI & $1.8 \pm 1.8$ \\
ASA I & $2(0.9 \%)$ \\
ASA II & $44(20 \%)$ \\
ASA III & $105(47.7 \%)$ \\
ASA IV & $52(23.6 \%)$ \\
\hline
\end{tabular}

$\mathrm{OA}=$ Osteoarthritis

$\mathrm{CCI}=$ Charlson Comorbidity Index

ASA $=$ American Society of Anesthesiologists

median time of pre-operative stay was 2 (1-36) days and median time of post-operative stay was 14 (1-50) days. Twenty-seven $(12.3 \%$ ) patients had post-operative complications or were readmitted to hospital within six months. Three (1.4\%) patients died within 12 months after their operation.

\section{Prevalence of PEM}

The prevalence of PEM in the patient cohort was $12.3 \%$ ( $n=$ 27). Pre-operative albumin levels $<3.5 \mathrm{~g} / \mathrm{dl}$ and TLC $\geq 1.5 \mathrm{~g} / 1$ were observed in $7(3.2 \%)$ patients, and pre-operative albumin levels $\geq 3.5 \mathrm{~g} / \mathrm{dl}$ and $\mathrm{TLC}<1.5 \mathrm{~g} / 1$ were observed in 108 $(49.1 \%)$ patients.

\section{Clinical characteristics of patients with PEM and without PEM}

Patients with PEM were significantly older (mean age $81.3 \pm 7.0, p<0.001)$, had a lower BMI $\left(24.7 \mathrm{~kg} / \mathrm{m}^{2} \pm\right.$ $4.1 \mathrm{~kg} / \mathrm{m}^{2}, p=0.022$ ) and showed more comorbid conditions (mean CCI 2.8 $\pm 2.0, p=0.002$ ) compared to patients without PEM (mean age 75.6 \pm 6.2 , mean BMI 26.8 \pm $4.3 \mathrm{~kg} / \mathrm{m}^{2}$, mean CCI: $\left.1.7 \pm 1.7\right)$. The median preoperative stay in the PEM group was 7 (1-36) days vs. 1 (1-22) day in the non PEM group $(p<0.001)$. No differences were found in median length of postoperative stay between the groups $(p<0.544)$. Three patients from the PEM group died within 12 months after surgery. Overall characteristics are presented in Table 2.
Table 2 Clinical characteristics according to PEM groups

\begin{tabular}{lllr}
\hline Variable & $\begin{array}{l}\text { Non-PEM } \\
n=193\end{array}$ & $\begin{array}{l}\text { PEM } \\
n=27\end{array}$ & $p$ value \\
\hline Age (years) & $75.6 \pm 6.2$ & $81.3 \pm 7.0$ & $<0.001$ \\
Gender & & & 0.390 \\
Female & $124(64.2 \%)$ & $20(74.1 \%)$ & \\
Male & $69(35.8 \%)$ & $7(25.9 \%)$ & \\
BMI & $26.8( \pm 4.3)$ & $24.7( \pm 4.1)$ & 0.022 \\
CCI & $1.7( \pm 1.7)$ & $2.8( \pm 2.0)$ & 0.002 \\
Diagnosis & & & \\
OA & $178(92.2 \%)$ & $17(63.0 \%)$ & 0.001 \\
Hip fracture & $15(7.8 \%)$ & $10(37.0 \%)$ & \\
ASA & & & 0.001 \\
Grade I & $2(100 \%)$ & 0 & \\
Grade II & $44(24.9 \%)$ & 0 & \\
Grade III & $93(52.5 \%)$ & $12(46.2 \%)$ & \\
Grade IV & $38(21.5 \%)$ & $14(53.8 \%)$ & \\
Pre-operative stay (days) & $1(1-22)$ & $7(1-36)$ & $<0.001$ \\
Length of post-operative stay (days) & $14(4-36)$ & $14(1-50)$ & $<0.544$ \\
\hline
\end{tabular}

ASA $=$ American Society of Anesthesiologists

$\mathrm{OA}=$ Osteoarthritis

\section{Association between PEM and post-operative complications}

In the PEM group $(40.7 \% ; n=11)$ significantly $(p<0.001)$ more patients had post-operative complications within three months compared with the non PEM group $(6.2 \% ; n=12)$. The morbidity rate within 6 months after surgery was $44.4 \%$ $(n=12)$ in patients with PEM and $7.8 \%(n=15)$ in patients without PEM $(p<0.001)$. Complications as causes for acute admission or re-admission in 220 patients are summarized in Table 3.

Table 3 Complications causing acute admissions or re-admissions within 6 months after surgery

\begin{tabular}{lll}
\hline Post-operative complications & Non-PEM $(n=193)$ & PEM $(n=27)$ \\
\hline Urinary complications & $3(1.6 \%)$ & $2(7.4 \%)$ \\
Pulmonary complications & $1(0.5 \%)$ & $5(18.5 \%)$ \\
Wound complications & $2(1 \%)$ & $2(7.4 \%)$ \\
Periprosthetic fractures & $2(1 \%)$ & 0 \\
Post-operative swelling & $2(1 \%)$ & 0 \\
Vascular complications & $1(0.5 \%)$ & $1(3.7 \%)$ \\
Septic arthritis of the hip & 0 & $1(3.7 \%)$ \\
Prosthetic infection & $1(0.5 \%)$ & 0 \\
Other complications & $3(1.6 \%)$ & $1(3.7 \%)$ \\
Total & $15(7.8 \%)$ & $12(44.4 \%)$ \\
\hline
\end{tabular}


In multivariate analysis, an increased risk for six month post-operative morbidity was observed for patients with PEM compared with the non PEM group (HR 6.3, 95\% CI: 1.7-23.1), adjusted for gender, age, type of diagnosis, time to operation, CRP, and HB. The Kaplan-Meier curves for postoperative morbidity between groups are shown in Fig. 1.

\section{Discussion}

PEM at hospital admission was present among $12.3 \%$ of patients. The aim of this retrospective study was to assess the prevalence of PEM and to investigate whether pre-operative nutritional parameters are associated with poor post-operative outcome in elderly patients undergoing primary total hip arthroplasty.

We could prove our hypothesis aiming that patients with PEM have inferior post-operative outcome after THA. We observed a higher risk for six month post-operative complications in patients with PEM compared with patients without PEM (HR 6.3, 95\% CI: 1.7-23.1). Furthermore, patients with PEM were significantly older, had a lower body mass index, and showed more comorbid conditions. Malnourished patients had a higher length of pre-operative stay. However, for the length of post-operative stay, no differences were observed between the groups.

The evaluated PEM prevalence $(12.3 \%)$ is not consistent with that reported by a related study [12]. Assessing PEM with same haematological markers of serum albumin and TLC, Nicholson et al. [12] investigated a rate of $30 \%$ in elective

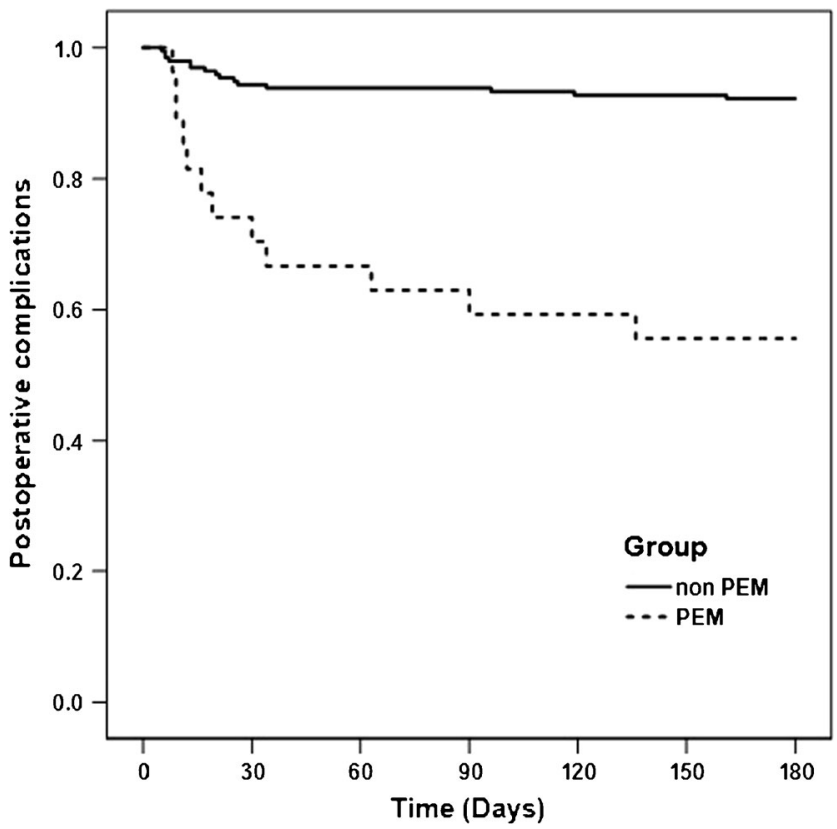

Fig. 1 Kaplan-Meier curves for post-operative morbidity between protein energy malnutrition (PEM) and no- protein energy malnutrition (non PEM) groups patients based on a smaller study population $(n=90)$ compared with our sample $(n=220)$. Our findings are similar to that of Huang et al. [6] where a prevalence of $8.5 \%$ was observed in patients undergoing elective joint arthroplasty. Investigations by Nicholson et al. [13] demonstrated a high rate $(60 \%)$ of malnutrition in hip fracture patients which is in accordance with our evaluation. PEM was observed in $8.7 \%$ $(17 / 195)$ of patients with OA but $40 \%(10 / 25)$ in hip fracture patients undergoing hip arthroplasty. In our study PEM on admission seems to be quite prevalent not only in older patients but also in hip fracture patients. This finding is supported by other clinical trials in orthopaedic patients [6]. Moreover several studies described negative consequences of malnutrition on mortality, morbidity, quality of life, or healthcare costs [2, 12, 14]. Recently, Lee et al. [2] identified low pre-operative albumin levels and hip surgery as negative predictors for home return in patients over the age of 85 .

However, a limitation of this study is that only a small number of patients had both albumin levels and TLC taken at admission. Out of 1183 initially screened operated patients, in $220(18.6 \%)$ both parameters were available. Serum albumin was not routinely collected at our department. Usually pre-operative laboratory records were determined a few weeks earlier through general practitioners or other institutions.

In this study we used biochemical nutritional markers to evaluate nutritional status as recommended to investigate PEM [3]. Because of the retrospective nature of the study we could not include more accepted and powerful tests such as Mini Nutritional Assessment (MNA) or Malnutrition Universal Screening Tool (MUST). According to other studies, the post-operative use of biochemical parameters is not recommended for clinical practice. Biochemical parameters are not reliable due to the fast decrease after acute illness or trauma [15]. We found that post-operatively low serum albumin and TLC occurred in $69.5 \%(146 / 210)$ of the patients regardless of their nutritional status at admission. The use of serum albumin as single variable evaluating PEM is insufficient. Using only one nutritional parameter could underestimate the prevalence of malnutrition in hospitalized patients [16]. However, our study used more than one nutritional parameter as indicator of malnutrition [3]. We additionally analyzed albumin and TLC values separately. Low albumin levels and normal TLC $\geq 1.5 \mathrm{~g} / 1$ were recorded for $7(3.2 \%)$ patients and a low TLC levels and normal albumin $\geq 3.5 \mathrm{~g} / \mathrm{dl}$ for $108(49.1 \%)$ patients.

Several studies demonstrated positive aspects of fast-track arthroplasty and indicated the importance of patient characteristics that could influence successful rehabilitation after fasttrack total hip or total knee arthroplasty [17]. In this context the nutritional status seems to play an important factor. Rudasill et al. created a pre-operative risk model to predict hypoalbuminemia at the patients undergoing THA. Individuals with three or more risk factors in the 7-point 
model were predicted to have hypoalbuminemia in $20.4 \%$ of the cases [18]. Consequently malnutrition at admission could represent a relevant exclusion criterion to perform fast-track surgery in affected patients. We have shown that basic laboratory values can predict risk for poor post-operative outcome in patients for elective surgery. Our results are comparable to that of $\mathrm{Lu}$ et al. [8] who examined a poor post-operative outcome in patients after a hip fracture surgery. Malnourished patients had an increased length of stay and showed a higher one year mortality rate compared with well-nourished patients. The results of the meta-analysis of seven cohort studies published by Tsantes et al. showed also that malnutrition is associated with higher infection rates after THA [19].

The used nutritional parameters seem to be an inexpensive method identifying surgical patients early at risk for poor outcome. Our findings highlight the importance of punctual nutritional assessment to detect dietary deficits. A simple preoperative assessment can additionally identify patients at risk. As a consequence, adequate dietary supplements can be provided and economic burden of readmissions may be avoided [20]. This is necessary, especially since low intake of calories and proteins, seems to be associated with low muscle mass which may result in sarcopenia. A Cochrane review of 41 studies showed that oral nutritional supplementation started before or soon after surgery can decrease complication rates in the first 12 months after hip fracture [21]. Vetrano et al. [22] underlines the need for nutritional support as muscle wasting can be decreased. Sarcopenia represents an important risk factor for adverse outcomes in the elderly [2, 20,23] like in our study. Sarcopenia is a screenable and optimizable risk factor, which needs further research. Our results showed that either normal weight or overweight does not exclude malnutrition, showing a mean BMI in the PEM group of $24.7 \pm 4.1 \mathrm{~kg} / \mathrm{m}^{2}$. Babu et al. evaluated the impact of the psoas-lumbar vertebral index of patients undergoing THA to be a risk factor for PJI [24]. Ryniecki et al. found the same results at revisional THA showing that patients having pre-operative hypoalbuminemia had an increased risk facing peri-operative complications, compared with those without hypoalbuminemia [25].

\section{Conclusion}

We observed in a large cohort a higher post-operative complication rate for malnourished patients undergoing elective THA compared with well-nourished patients. These results underline the importance of pre-operative nutritional assessment in older surgical patients. Serum albumin and TLC are inexpensive and valuable clinical markers of PEM with accurate prognostic evidence of post-operative outcomes. Further prospectively planned studies need to evaluate the impact of PEM and clinical outcome parameters together with the MNA or MUST. Clear guidelines need to be developed to determine cheap markers as a basis for pre-operative preventive treatment with oral supplements to decrease the incidence of post-operative complications.

Funding Open access funding provided by Medical University of Graz.

\section{Compliance with ethical standards}

Conflict of interest The authors declare that they have no conflicts of interest.

Ethics The regional Ethics Committee of the Medical University of Graz approved the study (Number: 27-021 ex 14/15).

Open Access This article is licensed under a Creative Commons Attribution 4.0 International License, which permits use, sharing, adaptation, distribution and reproduction in any medium or format, as long as you give appropriate credit to the original author(s) and the source, provide a link to the Creative Commons licence, and indicate if changes were made. The images or other third party material in this article are included in the article's Creative Commons licence, unless indicated otherwise in a credit line to the material. If material is not included in the article's Creative Commons licence and your intended use is not permitted by statutory regulation or exceeds the permitted use, you will need to obtain permission directly from the copyright holder. To view a copy of this licence, visit http://creativecommons.org/licenses/by/4.0/.

\section{References}

1. Pereira D, Peleteiro B, Araújo J, Branco J, Santos RA, Ramos E (2011) The effect of osteoarthritis definition on prevalence and incidence estimates: a systematic review. Osteoarthr Cartil 19: $1270-1285$

2. Lee B, Na S, Park M, Ham S, Kim J (2017) Home return after surgery in patients aged over 85 years is associated with preoperative albumin levels, the type of surgery, and APACHE II score. World J Surg 41:919-926

3. Li S, Zhang J, Zheng H, Wang X, Liu Z, Sun T (2019) Prognostic role of serum albumin, total lymphocyte count, and mini nutritional assessment on outcomes after geriatric hip fracture surgery: a metaanalysis and systematic review. J Arthroplast 34(6):1287-1296. https://doi.org/10.1016/j.arth.2019.02.003

4. Yi PH, Frank RM, Vann E, Sonn DA, Moric M, Valle CJD (2015) Is potential malnutrition associated with septic failure and acute infection after revision total joint Arthroplasty. Clin Orthop Relat Res 473:175-182

5. Bohl DD, Shen MR, Hannon CP, Fillingham YA, Darrith B, Della Valle CJ (2017) Serum albumin predicts survival and postoperative course following surgery for geriatric hip fracture. J Bone Joint Surg Am 99:2110-2118

6. Huang R, Greenky M, Kerr GJ, Austin MS, Parvizi J (2013) The effect of malnutrition on patients undergoing elective joint arthroplasty. J Arthroplast 28:21-24

7. Basu I, Subramanian P, Prime M, Jowett C, Levack B (2011) The use of biochemical parameters as nutritional screening tools in surgical patients. Surg Sci 2:89-94

8. Lu J, Chen Y, Zhang L, Wang X, Li Y, Wang C (2014) Laboratory nutritional parameters can predict one-year mortality in elderly patients with intertrochanteric fracture. Journal of Nutritional Health Food Science 2:1-6 
9. Koren-Hakim T, Weiss A, Hershkovitz A, Otzrateni I, Grosman B, Frishman S, Salai M, Beloosesky Y (2012) The relationship between nutritional status of hip fracture operated elderly patients and their functioning, comorbidity and outcome. Clin Nutr 31: 917-921

10. Charlson ME, Pompei P, Ales KL, MacKenzie CR (1987) A new method of classifying prognostic comorbidity in longitudinal studies: development and validation. J Chronic Dis 40:373-383

11. Lupei MI, Chipman JG, Beilman GJ, Oancea SC, Konia MR (2014) The association between ASA status and other risk stratification models on postoperative intensive care unit outcomes. Anesth Analg 118(5):989-994. https://doi.org/10.1213/ANE. 0000000000000187

12. Barker LA, Gout BS, Crowe TC (2011) Hospital malnutrition: prevalence, identification and impact on patients and the healthcare system. Int J Environ Res Public Health 8:514-527

13. Nicholson JA, Dowrick AS, Liew SM (2012) Nutritional status and short-term outcome of hip arthroplasty. J Orthop Surg (Hong Kong) 20:331-335

14. Lim SL, Ong KC, Chan YH, Loke WC, Ferguson M, Daniels L (2012) Malnutrition and its impact on cost of hospitalization, length of stay, readmission and 3-year mortality. Clin Nutr 31:345-350

15. So-Osman C, Nelissen R, Brand R, Brand A, Stiggelbout AM (2011) Postoperative anemia after joint replacement surgery is not related to quality of life during the first two weeks postoperatively. Transfusion $51: 71-81$

16. Kyle UG, Pirlich M, Schuetz T, Lochs H, Pichard C (2004) Is nutritional depletion by nutritional risk index associated with increased length of hospital stay? A population-based study. JPEN J Parenter Enteral Nutr 28:99-104

17. Husted H (2012) Fast-track hip and knee arthroplasty: clinical and organizational aspects. Acta Orthop Suppl 83:1-39

18. Rudasill S, Gittings DJ, Elkassabany NM, Liu J, Nelson CL, Kamath AF (2019) Preoperative risk factor score predicts malnutrition in total joint Arthroplasty patients. J Surg Orthop Adv 28(2): 97-103
19. Tsantes AG, Papadopoulos DV, Lytras T et al (2019) Association of malnutrition with periprosthetic joint and surgical site infections after total joint arthroplasty: a systematic review and meta-analysis. J Hosp Infect 103(1):69-77. https://doi.org/10.1016/j.jhin.2019.04. 020

20. Malafarina V, Reginster JY, Cabrerizo S, Bruyère O, Kanis JA, Martinez JA, Zulet MA (2018) Nutritional status and nutritional treatment are related to outcomes and mortality in older adults with hip fracture. Nutrients 30:555

21. Avenell A, Smith TO, Curtain J, Mak JCS, Myint PK. (2016) Nutritional supplementation for hip fracture aftercare in older people. Cochrane database of systematic reviews, issue 11. Art. No.: CD001880. DOI: https://doi.org/10.1002/14651858.CD001880. pub6

22. Vetrano DL, Landi F, Volpato S, Corsonello A, Meloni E, Bernabei R, Onder G (2014) Association of sarcopenia with short- and longterm mortality in older adults admitted to acute care wards: results from the CRIME study. J Gerontol A Biol Sci Med Sci 69:11541161

23. Landi F, Cruz-Jentoft AJ, Liperoti R, Russo A, Giovannini S, Tosato M, Capoluongo E, Bernabei R, Onder G (2013) Sarcopenia and mortality risk in frail older persons aged 80 years and older: results from ilSIRENTE study. Age Ageing 42:203-209

24. Babu JM, Kalagara S, Durand W, Antoci V, Deren ME, Cohen E (2019) Sarcopenia as a risk factor for prosthetic infection after total hip or knee arthroplasty. J Arthroplast 34(1):116-122. https://doi. org/10.1016/j.arth.2018.09.037

25. Rynecki ND, Congiusta DV, Fields M, Patel R, Vosbikian MM, Ahmed IH (2020) Increased risk of complications in patients with hypoalbuminemia undergoing revision total hip arthroplasty. J Orthop 21:253-257. https://doi.org/10.1016/j. jor.2020.03.006

Publisher's note Springer Nature remains neutral with regard to jurisdictional claims in published maps and institutional affiliations. 